RADIO SCIENCE, VOL. 37, NO. 1, 1004, 10.1029/2000RS002569, 2002

\title{
A new algorithm for high-quality ionogram generation and analysis
}

\author{
Feza Arıkan
}

Department of Electrical and Electronics Engineering, Hacettepe University, Ankara, Turkey

Orhan Arıkan

Department of Electrical and Electronics Engineering, Bilkent University, Ankara, Turkey

\author{
Sana Salous \\ Department of Electrical Engineering and Electronics, University of Manchester, Manchester, England, UK
}

Received 6 October 2000; revised 22 June 2001; accepted 30 July 2001; published 23 January 2002.

[1] Standard digital ionograms that are generated by fast Fourier transform or autoregressive modeling suffer from high interference levels due to other users of the HF channel which produce artifacts and distortion, hence complicating automatic processing and information extraction. In this paper, a new method is proposed to obtain high-quality ionograms of the desired layer reflections and automatically extract important information such as critical frequencies. Following the standard procedures, two sets of periodograms are obtained by using rectangular and Blackman windows. These two periodograms are filtered and fused utilizing an automatic edge-detection-based time-frequency detector. The fused ionogram provides sharp description of the layer reflections with very low sidelobe structure (ringing). The performance of this new ionogram algorithm is tested using chirp sounder data collected from an oblique midlatitude path. It is observed that the presented algorithm is highly successful in obtaining robust and sharp ionograms free of artifacts. Furthermore, a new algorithm is proposed for automated computation of dispersion and critical frequencies of the magnetoionic components detected on the ionogram. Since efficient signal-processing algorithms are utilized, the proposed method can be implemented in real time. INDEX TERMS: 6974 Radio Science: Signal processing, 6982 Radio Science: Tomography and imaging, 2494 Ionosphere: Instruments and techniques; KEYWORDS: ionosphere, ionosonde generation, ionosonde analysis, signal processing

\section{Introduction}

[2] Standard digital ionograms are an integral part of both technical and scientific studies, and they are utilized in a wide area ranging from automatic scaling to automatic link establishment [Goodman, 1992]. Ionograms that are generated from frequency-modulated continuous wave (FMCW or chirp) sounders usually use spectral analysis techniques such as the fast Fourier transform (FFT) algorithms or modern spectral analysis techniques such as autoregressive modeling. It is observed that these ionograms are cluttered with artificial artifacts and noise-like components that complicate

Copyright 2002 by the American Geophysical Union. 0048-6604/02/2000RS002569 automatic information extraction. Standard fast Fourier transforms (with rectangular windows), which are commonly used in ionogram generation, introduce undesired spectral leakage in the form of high sidelobes [Salous, 1997; Reinisch and Huang, 1983; Reinisch, 1986]. Examples of such ionograms (ionograms that are generated by standard FFT-based ionosondes) can easily be observed at many Internet sites such as those of the Millstone Hill digisonde, http://digisonde.haystack.edu/, the Tromsø digisonde, http://digisonde.phys.uit.no/, the Australian Space Forecast Centre, http://www.ips.gov. $\mathrm{au} / \mathrm{asfc} / \mathrm{current} / \mathrm{cbr}$.html, the University of Canterbury, http://www.phys.canterbury.ac.nz/ physion/ionogram. html, Institutet för Rymdfysik, http://www.irf.se/ ionogram/ionogram, Leibniz-Institut für Atmosphärenphysik, http://www.ionosonde.iap-kborn.de/ionogram, 
and the World Data Centre for Solar-Terrestrial Physics, Rutherford Appleton Laboratory, http://www.wdc.rl.ac.uk/ionosondes/, which all suffer from processing artifacts. Although the use of alternative windows such as the Blackman window partially eliminates the speckles due to spillovers, they also widen the sharp mainlobes of the rectangular-windowed peridogram [Salous, 1997, 1986; Fante, 1988].

[3] The obtained ionograms are usually scaled to obtain various parameters related to the characteristics of the ionosphere. In recent years, efforts are concentrated to automatize the manual scaling procedure to handle an increasing number of ionograms [Fox and Blundell, 1989; Gilbert and Smith, 1988; Igi et al., 1995; Poole and Mercer, 1995; Vugmeister and Radionov, 1995]. In the automatic scaling of digital ionograms the ionograms are generated by mimicking the steps taken by a manual scaler [Fox and Blundell, 1989]. Thus the first four stages of automatic scaling of ionograms (namely, cleaning the ionograms, organizing the signal echoes, forming continuous traces, and identifying layer traces) can be improved by introducing sharp and fast periodograms with a clean background.

[4] In this paper, a new method is proposed for highquality ionogram generation and magnetoionic component analysis. With the new ionogram algorithm the artificial artifacts due to hardware and processing of data are eliminated. By distortionless zooming capability, only the desired layer reflections or magnetoionic components can be observed on the ionogram. This capability facilitates the tracking of layers or modes. The data samples that are to be used in ionogram generation are processed by both sliding rectangular and sliding Blackman windows, and two sets of periodograms are obtained by discrete Fourier transform (DFT). These two periodograms are then separately median filtered along the time axis to partially eliminate the speckles. The zoomed-in, Blackman-windowed, and median-filtered ionogram is then passed through an edge detection filter that determines the significant and insignificant transitions of signal levels and eliminates insignificant edges. At the output of this filter, only the transitions around the desired signal components are retained. By making use of information on the significant edge locations, the two peridograms that are obtained by sliding rectangular and sliding Blackman windows are fused to form a sharp ionogram with negligible ringing. As a final step, a statistical thresholding algorithm is also used to partially eliminate noise like background. In the algorithms, various processing parameters, such as the length of the data sections to be processed by periodogram, the overlapping percent of sliding windows, the threshold in deciding significant and insignificant edges, and the threshold used in the fusing algorithm, are to be determined. It is observed that once these parameters are optimized for average midlatitude ionograms, the processing is quite robust and produces clean and sharp ionograms. To automatically extract important characteristics of the magnetoionic components, a second stage of processing is developed to measure the $6 \mathrm{~dB}$ frequency bandwidths, dispersion, and critical frequencies on the computed ionograms. The performance of the new ionogram generation and magnetoionic component analysis algorithm is demonstrated by using a chirp sounder data set collected from an oblique midlatitude path. The results are compared with those obtained by FFT and autoregressive (AR) processing presented by Salous [1997]. The proposed processing algorithm is fast enough to be implemented in real-time (online) applications.

[5] In section 2, the HF chirp sounder data set that is used in verification of the algorithm is described. In section 3, the new algorithm is introduced, and the algorithm is applied to the chirp sounder data to obtain example ionograms.

\section{Description of FMCW HF Chirp Sounder Data}

[6] The experimental data set used in the ionogram generation is collected by the wideband frequencymodulated continuous wave (FMCW) bistatic HF chirp sounder that was developed at the University of Birmingham [Salous, 1986]. The data set is obtained over a $273 \mathrm{~km}$ great circle path in the United Kingdom by a frequency sweep of $4 \mathrm{MHz}$ generated in $4 \mathrm{~s}$ with a sweep separation of $10 \mathrm{~s}$. The data were sampled with a 12 bit analog to digital converter at a sampling rate of $16,384 \mathrm{~Hz}$, giving a total of 65,536 samples per sweep. A typical sweep period is presented in Figure 1 , which shows the digitized signal level versus the sweep time in seconds. It can be seen from the figure that superimposed on the fading envelope of the received signal is a high level of interference that is usually present in the HF band. Since the ionograms are obtained by dividing the received signal into sections prior to the spectral analysis, the effect of interference is to raise the noise floor in the section where it occurs, as can be seen from Figures $2 a$ and 2b. The effect of interference can be reduced by 


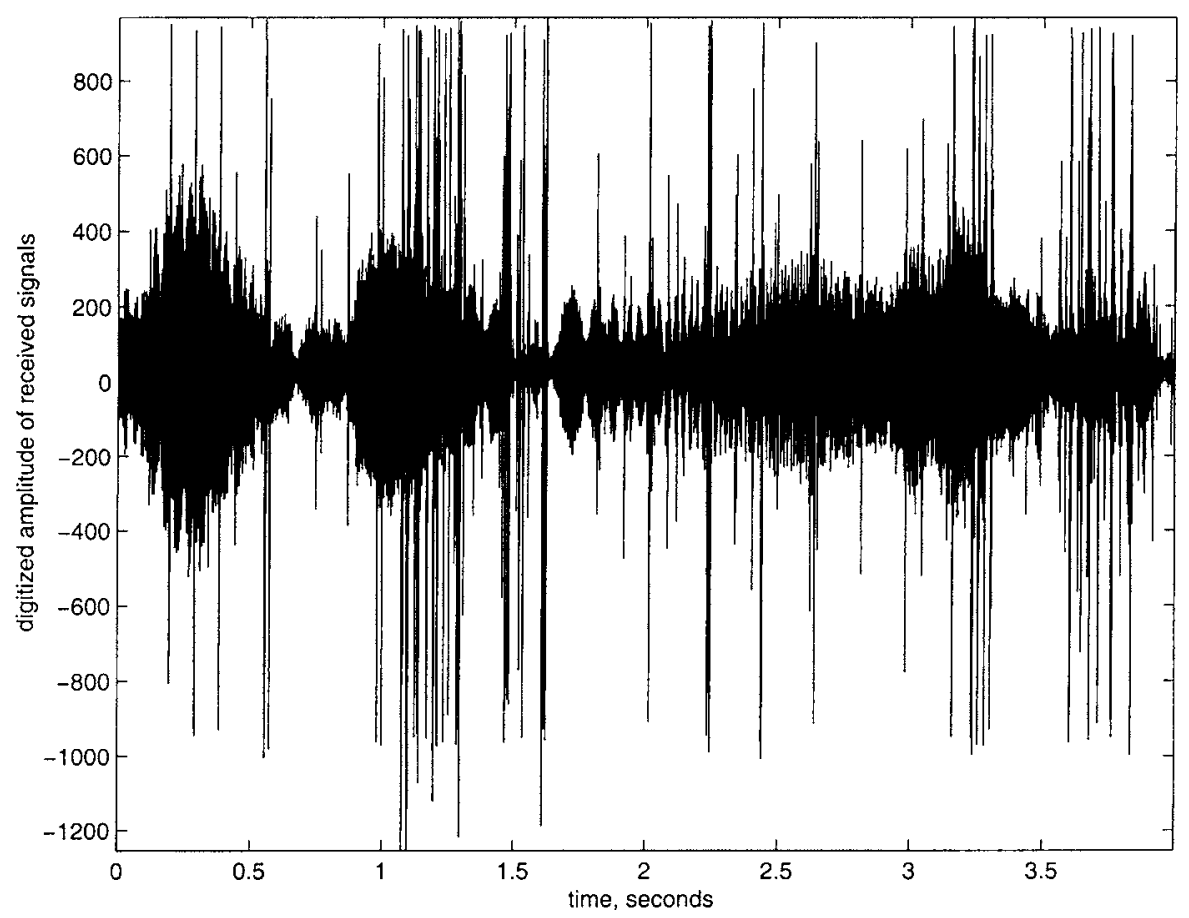

Figure 1. Received data samples in a frequency sweep.

clipping its level to a value equal to or higher than the peak of the signal [Salous, 1997].

\section{Proposed Method for Generation and Analysis of Ionograms}

[7] A short discussion of the ionogram generation algorithm was presented by Arlkan et al. [2000a, $2000 \mathrm{~b}]$, and the framework is summarized in Figure 3. Let $\mathbf{s}=\left[\begin{array}{llll}s_{0} & s_{1} & \cdots & s_{N_{d}}\end{array}\right]^{T}$ be the received signal vector in one frequency sweep, where $N_{d}$ is the total number of signal samples in that period and the superscript $T$ denotes the transpose. The first goal is to generate a clear ionogram to observe the reflections from layers and resolve the magnetoionic components. To capture the variation of the reflected signal characteristics as a function of frequency, short data sequences $\mathbf{s}_{k}$ of length $N_{w}$ will be extracted from the signal vector $\mathbf{s}$ with an allowance of $N_{o v}$ overlapping samples as

$$
\mathbf{s}_{k}=\left[s_{(k-1) N_{o v}+1} \cdots s_{(k-1) N_{o v}+N_{w}}\right]^{T},
$$

where $k=1,2, \ldots, N_{k}$ and $N_{k}=\left(N_{d}-N_{w}+N_{o v}\right) / N_{o v}$. The standard procedure in obtaining ionograms is to apply a fast Fourier transform (FFT) on the extracted data samples and, subsequently, arrange the sequences in the time-frequency plane [Salous, 1986; Fox and Blundell, 1989; Lynn, 1998; Reinisch et al., 1997; MacDougall et al., 1995]. The use of $\mathbf{s}_{k}$ with no further windowing provides sharp ionograms with significant sidelobes. To reduce the effects of sidelobes on the detection of echoes, other windows such as the Blackman window are used (its first sidelobe level is $57 \mathrm{~dB}$ down from the mainlobe). However, the mainlobe beam width of the Blackman window is 3 times that of the rectangular window. Thus it is desirable to combine the best of these two window functions, i.e., a sharp mainlobe and low spillovers. For this end, in the proposed approach, ionograms are computed for both the rectangular- and Blackmanwindowed sequences:

$$
\begin{aligned}
\mathbf{s}_{k R}= & {\left[s_{(k-1) N_{o v}+1} w_{R}(0)\right.} \\
& \left.\cdots s_{(k-1) N_{o v}+N_{w}} w_{R}\left(N_{w}-1\right)\right]^{T}, \\
\mathbf{s}_{k R}= & {\left[s_{(k-1) N_{o v}+1} w_{B}(0)\right.} \\
& \left.\cdots s_{(k-1) N_{o v}+N_{w}} w_{B}\left(N_{w}-1\right)\right]^{T},
\end{aligned}
$$

where $w_{R}(n)$ and $w_{B}(n)$ are given in Table 1.

[8] In transforming the windowed signal sequences into the frequency domain, a chirp z-transform algorithm that provides uniformly spaced frequency samples in an 

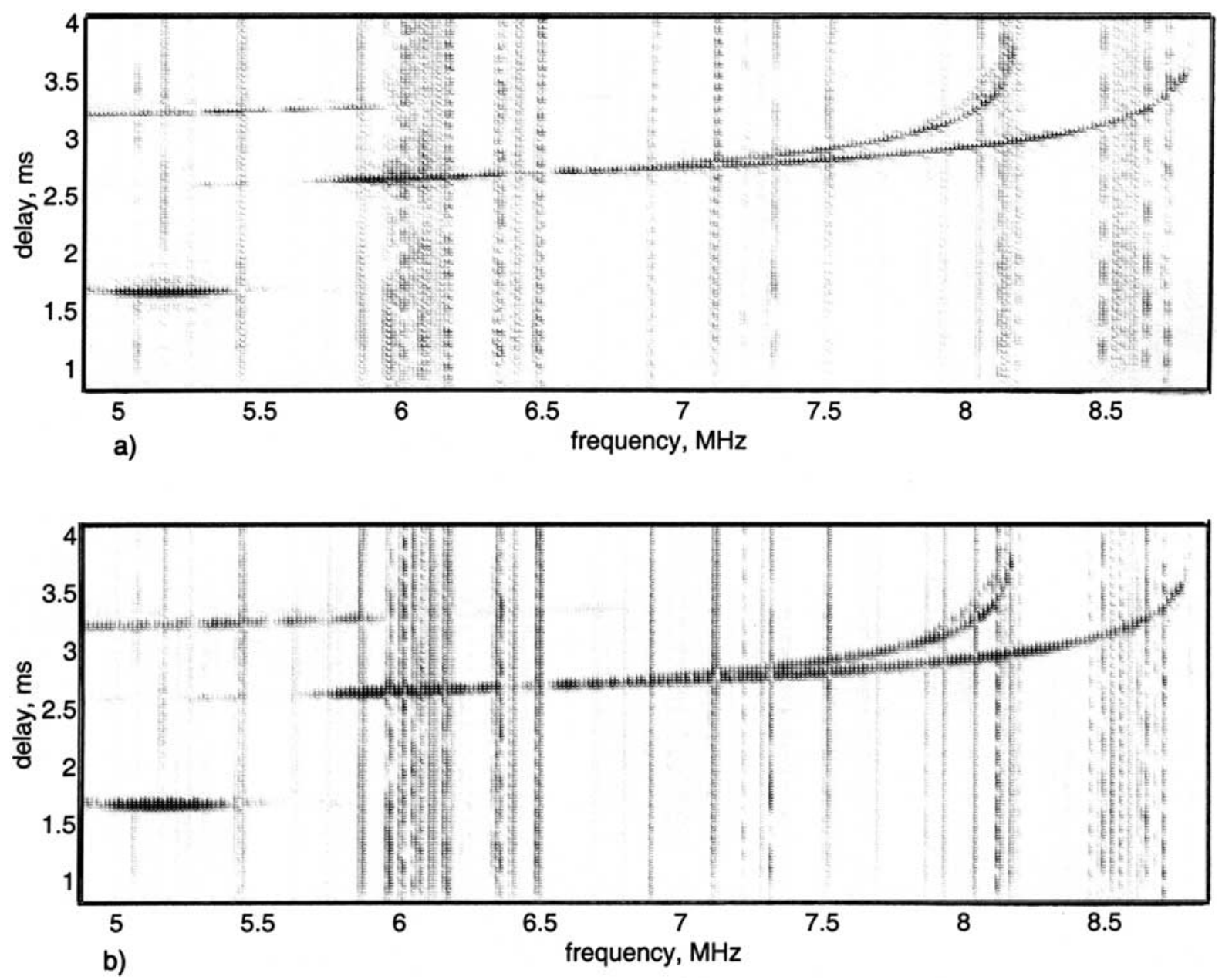

Figure 2. (a) Rectangular-windowed ionogram cluttered by spectral spillovers. (b) Blackman-windowed ionogram with a widened mainlobe, which reduces the sharpness.

interval $\left[f_{1}, f_{2}\right]$ is used [Fante, 1988]. By choosing the interval $\left[f_{1}, f_{2}\right]$ as a short interval containing the desired reflections, a high-resolution investigation of the spectral domain response can be achieved with less computation than is involved in the classical FFT-based analysis [Fante, 1988]. In the following presentation the computed ionograms in the frequency interval $\left[f_{1}, f_{2}\right]$ will be referred to as the "zoomed-in" ionogram. The chirp $z$-transformation of the data sequence $\mathbf{s}_{k}$ is defined as

$$
\begin{aligned}
S_{k_{; f 1, f 2}}(m)= & \exp \left[-j \frac{\pi}{N}\left(f_{2}-f_{1}\right)\left(m-N_{w}\right)^{2}\right] \\
& \cdot \sum_{n=0}^{N_{w}-1} e^{-j 2 \pi f_{1} n} \exp \left[-j \frac{\pi}{N}\left(f_{2}-f_{1}\right) n^{2}\right] s_{k}(n) \\
& \cdot \exp \left[j \frac{\pi}{N}\left(f_{2}-f_{1}\right)(m-n-N)^{2}\right],
\end{aligned}
$$

where $N<N_{w}$ is the number of computed frequency domain samples in the interval $\left[f_{1}, f_{2}\right]$. The required computation of $S_{k_{f_{1}, f_{2}}}(m)$ can be efficiently performed using FFT techniques as detailed by Fante [1988]. Typically, the $\left[f_{1}, f_{2}\right]$ interval remains the same for all sections $k$. Therefore, for notational simplicity, we will call the transformed sections $S_{k}(m)$. For both the rectangular- and Blackman-windowed sections of (2) and (3) the zoomed-in ionograms are formed as the following matrices:

$$
\begin{aligned}
& \mathbf{S}_{R}=\left[S_{1 R} \cdots S_{k R} \cdots S_{N_{k} R}\right], \\
& \mathbf{S}_{B}=\left[S_{1 B} \cdots S_{k B} \cdots S_{N_{k} B}\right],
\end{aligned}
$$

where $S_{k R}$ and $S_{k B}$ are the column vectors formed by the computed chirp z-tranforms of the weighted sections $s_{k R}$ and $s_{k B}$, respectively. 


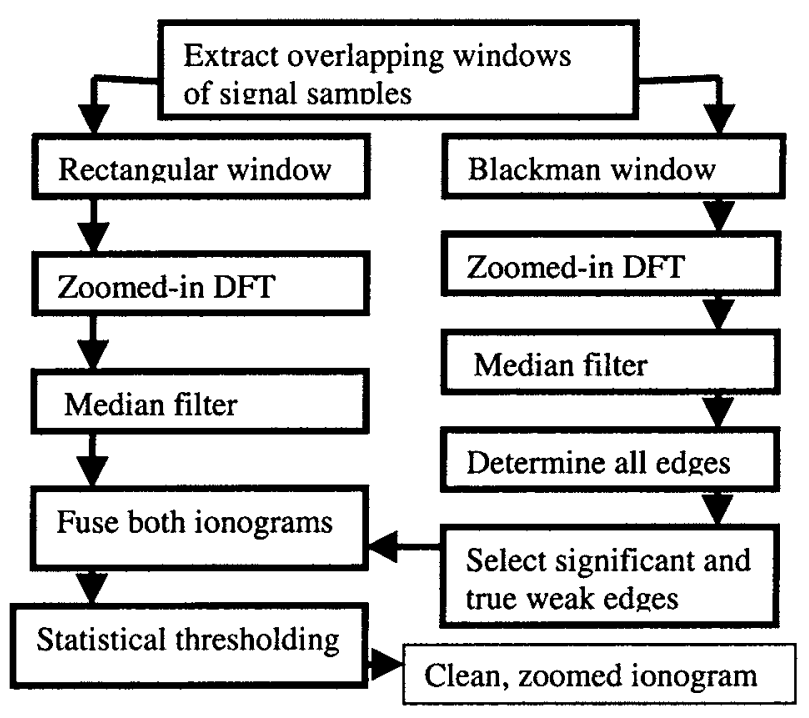

Figure 3. Flowchart of the new ionogram generation algorithm.

[9] Examples of ionograms obtained by the rectangular window and the Blackman window computed over the full range of frequencies as in (4) are presented in Figures $2 \mathrm{a}$ and $2 \mathrm{~b}$, respectively. Note that the frequency interval of $\left[f_{1}, f_{2}\right]$ is mapped to its corresponding time delay in the figures. In Figure 2a, rectangular windowing causes artificial artifacts due to spillovers. Similar artifacts can readily be observed in other FFT-based ionograms such as those of Lynn [1998] and Reinisch et al. [1997]. In Figure 2b it is observed that by Blackman windowing, the artifacts due to FFT are reduced but the sharp mainlobe of the ionogram is widened.

[10] We would like to obtain the sharpness of the rectangular-windowed ionogram and low spillovers of the Blackman-windowed ionogram at the same time and free the ionogram from all artifial artifacts and noise-like components. So, in the second step of the ionogram generation, both $S_{R}$ and $S_{B}$ ionograms are median filtered with a filter length $N_{m}$, along the time axis.

$$
\begin{aligned}
S_{k R}(m)= & \operatorname{median}\left\{S_{\left(k-\left(N_{m}-1\right) / 2\right) R}(m),\right. \\
& \left.\cdots, S_{\left(k+\left(N_{m}-1\right) / 2\right) R}(m)\right\}, \\
S_{k B}(m)= & \operatorname{median}\left\{S_{\left(k-\left(N_{m}-1\right) / 2\right) B}(m),\right. \\
& \left.\cdots, S_{\left(k+\left(N_{m}-1\right) / 2\right) B}(m)\right\},
\end{aligned}
$$

In this way, the ionograms are smoothed, and the effect of unwanted noise-like components is reduced.

[11] In the third step of ionogram generation, significant edges in the median-filtered and zoomed-in ionograms are determined by using Canny's method of edge detection [Canny, 1986]. Canny's method is widely used by the image-processing community, and it is successfully standardized in the MATLAB imageprocessing toolbox. In order to find the significant edges, Canny's method searches for the local maxima of the gradient of the periodogram. The gradient is calculated using the derivative of a Gaussian filter. This method uses two thresholds to detect strong and weak edges, and weak edges are included in the output only if they are connected to strong edges. Thus Canny's method of edge detection is quite robust to noise components and is highly successful in detecting true weak edges. The advantage of using this technique is illustrated in Figure 4, which shows the difference between all-edges detection (Figure 4a) and the true weak edges and strong edges (Figure $4 b$ ). Figure $4 a$ is obtained by the imaging routines of MATLAB, and it is totally computer generated. It shows all significant and weak edges in the ionogram. Figure $4 \mathrm{~b}$ denotes the same ionogram after it is processed by Canny's edge detection method. This time, the imaging routines indicate only the true weak edges and strong edges in the ionogram.

[12] After determining the locations of significant signal transitions with the edge detection algorithm, median-filtered Blackman- and rectangular-windowed spectrum estimates are fused to obtain a sharp periodogram with negligible ringing. The fusion process is implemented in three stages: initialization, reassignment,

\begin{tabular}{|c|c|c|c|}
\hline $\begin{array}{l}\text { Window } \\
\text { Function }\end{array}$ & $0 \leq n \leq N_{w}-1$ & $\begin{array}{l}\text { First Null } \\
\text { Beam Width }\end{array}$ & $\begin{array}{c}\text { Sidelobe Peak } \\
\text { Relative to Mainlobe }\end{array}$ \\
\hline $\begin{array}{l}\text { Rectangular } w_{R}(n) \\
\text { Blackman } w_{B}(n)\end{array}$ & $0.42-0.5 \cos \left(\frac{2 \pi n}{N w}\right)+0.08 \cos \left(\frac{4 \pi n}{N w}\right)$ & $\begin{array}{l}2 / N_{w} \\
6 / N_{w}\end{array}$ & $\begin{array}{l}-13.5 \mathrm{~dB} \\
-57 \mathrm{~dB}\end{array}$ \\
\hline
\end{tabular}
and statistical thresholding. In the first stage the fused spectrum is initialized with the Blackman-windowed

Table 1. Comparison of Rectangular Window and Blackman Window 


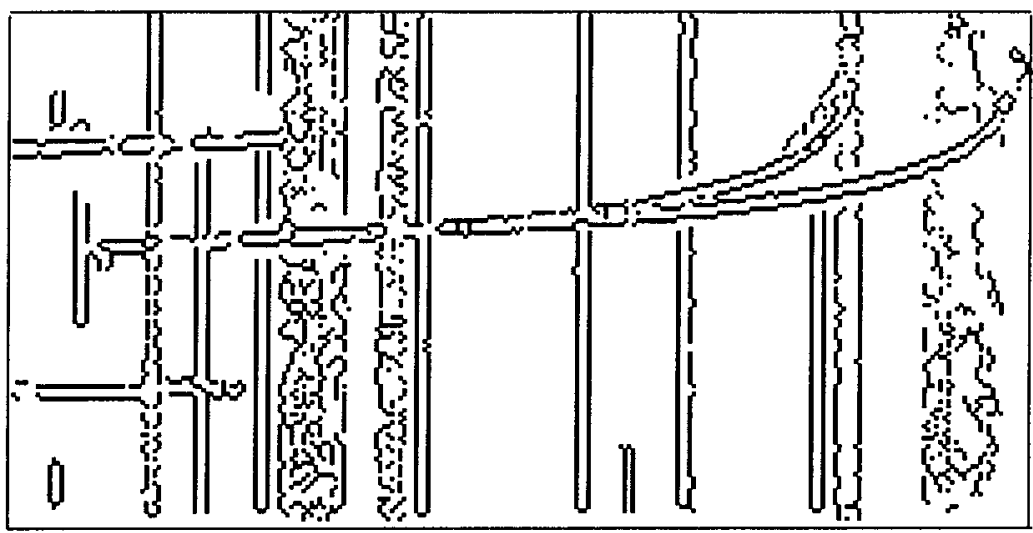

a)

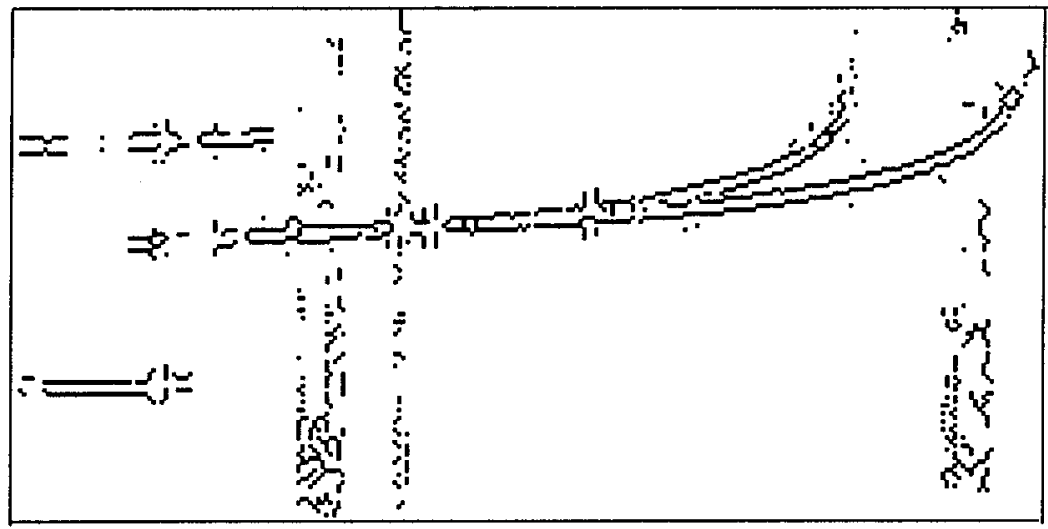

b)

Figure 4. (a) All edges in the ionogram detected by the imaging routines of MATLAB. (b) True weak and significant edges obtained when the ionogram is processed by Canny's method.

spectrum. This way, the fused spectrum acquires its lowringing feature. However, the spectral peaks in the ionograms are not as sharply resolved as those in the rectangular-windowed spectral estimates. Therefore, in the second stage of the fusion process, values of those pixels that are in the close proximity of the previously detected significant edges are reassigned with the corresponding pixel values of the rectangular-windowed spectral estimate. In this way, the desired features of both the Blackman- and rectangular-windowed spectral estimates are captured in the fused spectrum. In the final stage the obtained fused spectrum values are thresholded by using a time-dependent threshold value to zero out those parts of the spectrum with statistically insignificant power levels. The threshold is chosen on the basis of the mean and standard deviation of the fused spectrum magnitude distribution. Figure 5a presents a sharp and clean fused ionogram, and in Figure $5 \mathrm{~b}$ the ionogram is zoomed in on the desired $1 F_{2}$ layer ordinary and extraordinary waves.

[13] The success of this new ionogram algorithm is mostly determined by the proper choice of parameters, such as the window length $N_{w}$, the number of overlapping samples $N_{o v}$, the median filter length $N_{m}$, and the threshold levels used in determining the significant and insignificant signal transitions and statistical thresholding routine. Most notably, $N_{w}$ should be chosen so that a well-balanced result between the time and frequency resolution of the components is achieved. An appropriate 

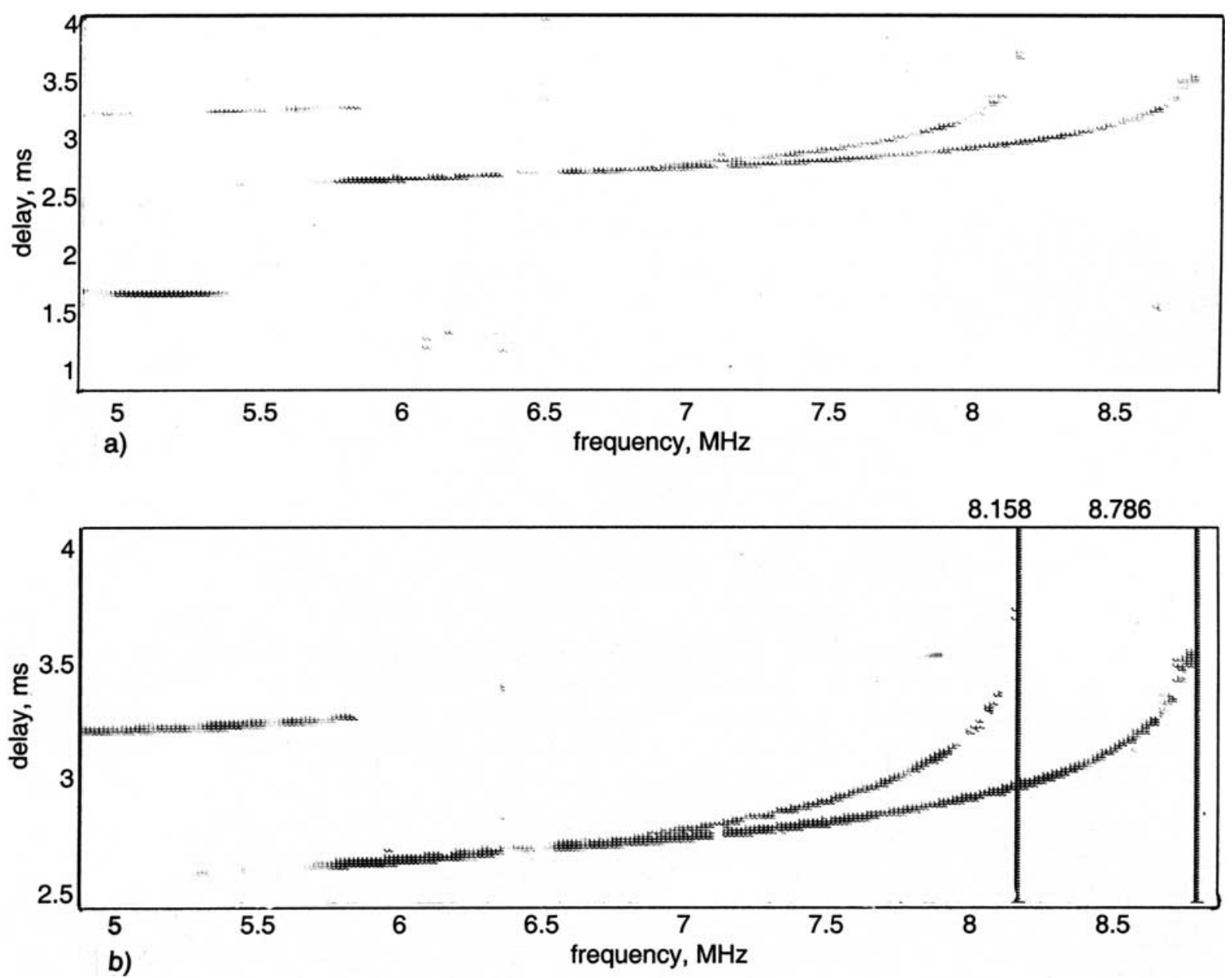

Figure 5. (a) Clean and sharp ionogram after fusing and statistical thresholding is applied. (b) Zoomed-in ionogram to observe $F$ layer. The critical frequencies of the ordinary and extraordinary wave components are indicated on the ionogram.

choice of $N_{w}$ is 512, which provides a reasonable tradeoff between the time and frequency resolution of the desired reflections in the investigated ionograms. Generally, once $N_{w}$ is properly chosen, the ionograms on that specific link are quite robust to variations in the ionospheric characteristics, and its value does not need any modifications within the link. The number of overlapping samples $N_{o v}$ is set to 256 , which corresponds to $50 \%$ overlap between successive sections.

[14] The thresholds in the edge detection and statistical thresholding routines are also set on the basis of the results of an off-line investigation of the recorded ionograms. Once they are set, the same thresholds can be reliably used in processing the new ionograms acquired from the same link. Furthermore, as shown in Figures $5 \mathrm{a}$ and $5 \mathrm{~b}$, statistical thresholding algorithms eliminate the leftover insignificant artifacts. In an extensive set of investigations we have observed that once the parameters are set in a quasi-optimum manner, the ionogram algorithm is very robust to variations in the ionospheric conditions, and high-quality ionograms are generated.

[15] Determination of group path delay, $6 \mathrm{~dB}$ dispersion bandwidth, direction finding, and real height analysis from measurements of ionosondes plays an important role in channel characterization [Salous, 1997, 1986, 1994; Goodman, 1992; Fox and Blundell, 1989; Black et al., 1993; Reilly and Kolesar, 1989; Lynn, 1998]. However, current scaling algorithms cannot provide all the parameters, and they are mostly operated manually (or at least require operator intervention) [Poole and Mercer, 1995; Vugmeister and Radionov, 

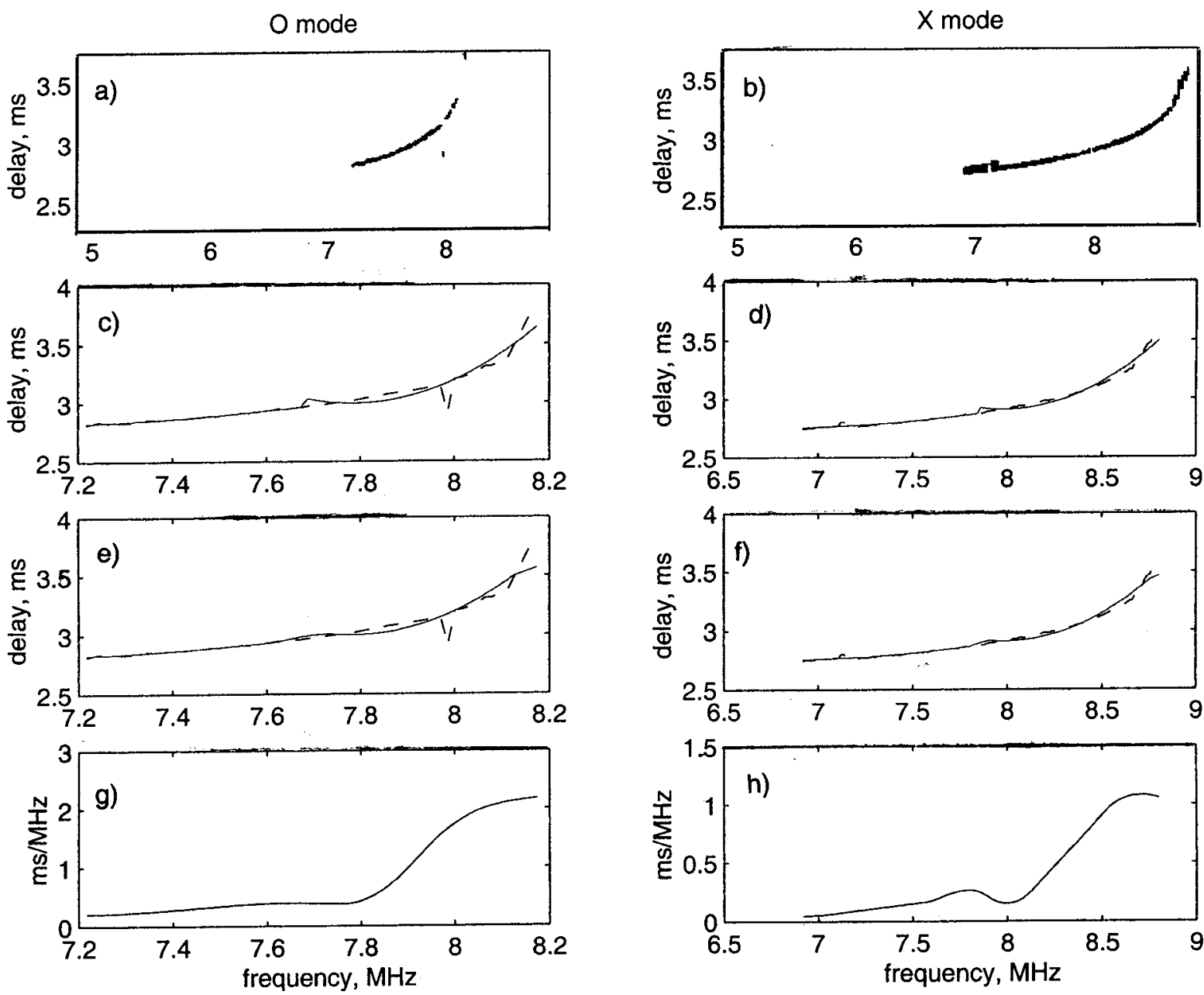

Figure 6. (a and b) Masked $O$ and $X$ modes, respectively; (c and d) original data (dashed curves) with interpolated data (solid curves) for $O$ and $X$ modes, respectively; (e and f) original data (dashed curves) with smoothed interpolated data (solid curves) for $O$ and $X$ modes, respectively; ( $\mathrm{g}$ and $\mathrm{h}$ ) slope of group time delay curve in $\mathrm{ms} / \mathrm{MHz}$.

1995]. In the rest of this section, we present novel processing algorithms for automated analysis of the obtained clean and sharp ionograms. These algorithms can easily be implemented into hardware and improve present routines such as that of Gilbert and Smith [1988]. Determination of group delay corresponding to each magnetoionic component in the generated ionogram is done as follows: First, we determine the frequency at which the ordinary and extraordinary waves are separated by automatically searching the maximum delays occuring at each frequency. Once the separation frequency is approximately obtained, two masks corresponding to each observed mode are constructed. Then, using the constructed masks, as shown in Figures 6a and 6b, the $O$ and $X$ modes are automatically extracted out of the ionogram in the frequency region above the separation frequency. The frequency interval is divided into two sections of equal length, and a quadratic fit in each section to each of the masked modes is performed to obtain a polynomial approximation to $O$ and $X$ modes beyond the separation frequency (Figures $6 \mathrm{c}$ and $6 \mathrm{~d}$ ). A sliding averaging filter is used to smooth out the discontinuity of the two polynomial fits at the junction of the two sections (Figures 6e and 6f). The smoothed polynomial approximations of $O$ and $X$ modes are the estimated group time delay of each component. These estimates are also used in the computation of other features of the $O$ and $X$ modes as explained below. All 
Table 2. Comparison of Spectral Widths of $O$ and $X$ modes with AR Processing Given by Salous [1997]

\begin{tabular}{lccccc}
\hline & \multicolumn{2}{c}{$O$ Mode } & \multicolumn{2}{c}{$X$ Mode } \\
\cline { 2 - 3 } \cline { 5 - 6 } & New Algorithm & AR & & New Algorithm & AR \\
\hline$B T \leq 3$ & $84.7 \%$ & $70 \%$ & & $84.09 \%$ & $57 \%$ \\
$B T \leq 5$ & $86.24 \%$ & $\cdots$ & $84.71 \%$ & $\cdots$ \\
\hline
\end{tabular}

of the steps in the processing are completed automatically with MATLAB routines, and they are free of operator intervention.

[16] Dispersion of chirp pulses by the ionosphere has been investigated in detail by Salous [1994], and the time-bandwidth product was obtained as [Salous, 1994, equation (19a)]

$$
B T(f)=\frac{\partial \tau_{g}}{\partial f} B^{2},
$$

where $\partial \tau_{g} / \partial f$ is the slope of group time delay and $B$ is the frequency range swept in $T$ seconds. $B T(f)$ of $O$ and $X$ modes can be automatically obtained by a numerical approximation of the derivative of corresponding group time delay curves as shown in Figures $6 \mathrm{~g}$ and $6 \mathrm{~h}$. More robust estimates to the derivatives are obtained automatically by using a sliding averaging filter on the numerically computed derivative curves.

[17] The $B T$ values can also be used as an indication of the spectral width [Salous, 1997]. In order to compare the spectral widths obtained by Salous [1997] with those estimated from the $B T$ algorithm, the $B T$ algorithm was applied to the same set of 90 sweeps. The percentages of occurrence of the spectral widths, i.e., $B T \leq 3$ and $B T \leq$ 5 , are tabulated in Table 2 with those obtained from the AR processing [Salous, 1997]. It is observed that the spectral widths from the $B T$ algorithm are considerably narrower than those obtained from AR analysis, indicating a sharper peak. However, it is important to note that the frequency resolution employed in the present study is finer than that reported by Salous [1997] (15 kHz versus $125 \mathrm{kHz}$ ). Hence the results of Table 2, although indicative of sharper peaks expected from the new algorithm, should be compared with caution.

[18] Another important feature of the ionograms is the critical frequencies of the observed magnetoionic components. In this paper, we introduce a numerical procedure that can be used to automatically compute the desired critical frequencies, $f_{\mathrm{o}} \mathrm{f}_{2}$ and $f_{\mathrm{x}} \mathrm{f}_{2}$, on the basis of the polynomial fit obtained for each mode. A similar automated procedure can be implemented for $E$ layer parameters. For each of the magnetoionic components the critical frequecies can be obtained by fitting a function in the form of $\alpha /\left(f_{i}-f\right)$ to the polynomial approximation curve of the group time delay shown in Figures $6 \mathrm{e}$ and $6 \mathrm{f}$. The fitting parameters $\alpha$ and $f$ are obtained as the minimizers of

$$
J(a, f)=\sum_{i=1}^{N}\left(y_{i}-\frac{\alpha}{f_{i}-f}\right)^{2} .
$$

The minimizers of the above cost function in terms of $f$ can be obtained as the minimizer of

$$
D(f)=\left|\begin{array}{cc}
\sum_{i=1}^{N} \frac{y_{i}}{f_{i}-f} & \sum_{i=1}^{N} \frac{y_{i}}{\left(f_{i}-f\right)^{2}} \\
\sum_{i=1}^{N} \frac{1}{\left(f_{i}-f\right)^{2}} & \sum_{i=1}^{N} \frac{1}{\left(f_{i}-f\right)^{3}}
\end{array}\right| .
$$

By a simple one-dimensional search, the minimizer of $D(f)$ can be obtained giving the critical frequency.

[19] Over the 90 sweeps, $f_{o} f_{2}$ and $f_{x} f_{2}$ values are computed and plotted in Figure 7. As an example, the computed $f_{o} f_{2}$ and $f_{x} f_{2}$ values are indicated on the zoomed-in ionogram shown in Figure $5 \mathrm{~b}$.

[20] The MATLAB codes for the ionogram generation algorithm and the automatic information extraction for the computation of dispersion, $6 \mathrm{~dB}$ bandwidth, and critical frequencies for one sweep take less than a minute, approximately, on a PC with Pentium processor. Thus the computation time for the algorithm is short enough for it to be used in a real-time application. When compared with current ionogram generation and scaling routines (such as those of Gilbert and Smith [1988], Reinisch et al. [1997], Vugmeister and Radionov [1995], and Igi et al. [1995]), the suggested algorithm is faster and more accurate.

\section{Conclusions}

[21] In this paper, a new method is presented to obtain high-quality ionograms and automatically extract 


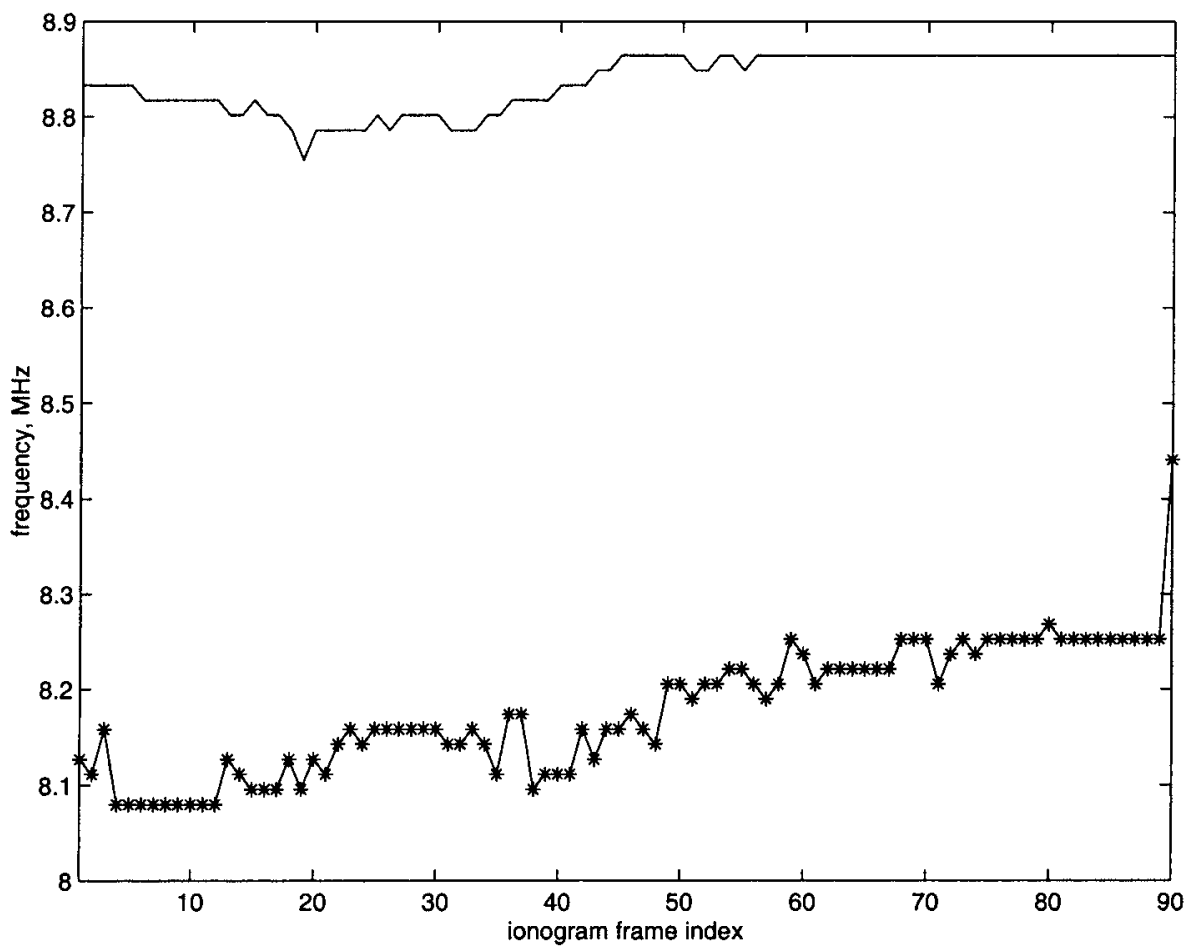

Figure 7. Critical frequencies for $O$ and $X$ modes for 90 sweeps: The solid curve with stars denotes $f_{a} f_{2}$ values, and the solid curve without stars denotes $f_{x} f_{2}$ values.

important information on the observed magnetoionic components. In the generation of the ionograms the data samples that are processed by both a sliding rectangular window and a sliding Blackman window are processed with the chirp z-transform (i.e., zoomedin DFT) to obtain two sets of spectograms. These two spectograms are separately median filtered and fused to obtain a sharp periodogram with negligible ringing. Fusing is based on an edge detection routine that automatically enhances significant transitions near desired signal components and eliminates insignificant transitions due to artifacts. A statistical thresholding algorithm is also used to eliminate remaining noise-like background. The performance of this new ionogram algorithm is verified with chirp sounder data collected from an oblique midlatitude path. It is observed that the presented algorithm is highly successful in obtaining robust and sharp ionograms free of artifacts and processing noise. This algorithm is also fast enough to be used in real-time (online) applications. As demonstrated on the investigated data set, the generated ionograms can be successfully used for automatic information extraction such as critical frequencies of ionospheric layers and for estimation of channel dispersion. It is observed that the new ionogram generation algorithm leads to higher resolution ionograms than ionograms obtained by FFT or AR methods.

\section{References}

Arıkan, F., O. Arıkan, and S. Salous, A new algorithm for computation of digital ionograms, paper presented at International Symposium on Equatorial Aeronomy, Air Force Off. of Sci. Res., Antalya, Turkey, May 2000a.

Arıkan, F., S. Salous, and O. Arıkan, A new algorithm for high quality ionograms, Electron. Lett., 36, 985-987, 2000b.

Black, Q. R., J. F. Wood, Jr., A. G. Sonsteby, and W. M. Sherill, A direction finding ionosonde for ionospheric propagation research, Radio Sci., 28(3), 795-809, 1993.

Canny, J., A computational approach to edge detection, IEEE Trans. Pattern Anal. Mach. Intel., 8, 679-698, 1986.

Fante, R. L., Signal Analysis and Estimation, John Wiley, New York, 1988.

Fox, M. W., and C. Blundell, Automatic scaling of digital ionograms, Radio Sci., 24(6), 747-761, 1989.

Gilbert, J. D., and R. W. Smith, A comparison between the automatic ionogram scaling system ARTIST and the standard manual method, Radio Sci., 23(6), 968-974, 1988.

Goodman, J. M., HF Communications-Science and Technology, Van Nostrand Reinhold, New York, 1992. 
Igi, S., K. Nozaki, M. Nagayama, A. Ohtani, H. Kato, and K. Igarashi, Automatic ionogram processing systems in Japan, in Ionosonde Networks and Stations, Rep. UAG-104, edited by P. Wilkinson, World Data Cent. A for Sol.-Terr. Phys., Boulder, Colo., 1995.

Lynn, K. J. W., Oblique sounding in Australia, URSI INAG Ionospheric Stn. Inf. Bull. 62, pp. 477,-492, Ionospheric Network Adv. Group, Int. Counc. of Sci. Unions, Paris, 1998.

MacDougall, J. W., I. F. Grant, and X. Shen, The Canadian advanced digital ionosonde: Design and results, in Ionospheric Networks and Stations, Rep. UAG-104, edited by P. Wilkinson, World Data Cent. A for Sol.-Terr. Phys., Boulder, Colo., 1995.

Poole, A. W. V., and C. C. Mercer, Towards a semi-automatic scaling ionogram to provide model parameters rather than traditional scaling parameters, in Ionospheric Networks and Stations, Rep. UAG-104, edited by P. Wilkinson, World Data Cent. A for Sol.-Terr. Phys., Boulder, Colo., 1995.

Reilly, M. H., and J. D. Kolesar, A method for real height analysis of oblique ionograms, Radio Sci., 24(4), 575583, 1989.

Reinisch, B. W., New techniques in ground-based ionospheric sounding and studies, Radio Sci., 21(3), 331-341, 1986.

Reinisch, B. W., and X. Huang, Automatic calculation of electron density profiles from digital ionograms, Radio Sci., 18(3), 477-492, 1983.
Reinisch, B. W., D. M. Haines, K. Bibl, I. Galkin, X. Huang, D. F. Kitrosser, G. S. Sales, and J. L. Scali, Ionospheric sounding in support of over-the-horizon radar, Radio Sci., 32(4), 1681-1694, 1997.

Salous, S., FMCW channel sounder with digital processing for measuring the coherence of wideband HF radio links, IEE Proc., F, Commun. Radar Signal Process., 133, 456-462, 1986.

Salous, S., Dispersion of chirp pulses by the ionosphere, $J$. Atmos. Terr. Phys., 56, 979-994, 1994.

Salous, S., On the potential applicability of auto-regressive spectral estimation to HF chirp sounders, J. Atmos. Terr. Phys., 59, 1961-1972, 1997.

Vugmeister, B. O., and V. V. Radionov, A semi-automatic interpretation of ionograms, in Ionospheric Networks and Stations, Rep. UAG-104, edited by P. Wilkinson, World Data Cent. A for Sol.-Terr. Phys., Boulder, Colo., 1995.

F. Arıkan, Elektrik ve Elektronik Müh. Bölümü, Hacettepe Üniversitesi, Beytepe 06532, Ankara, Turkey. (arikan@, hacettepe.edu.tr)

O. Arıkan, Elektrik ve Elektronik Müh. Bölümü, Bilkent Üniversitesi, Bilkent 06533, Ankara, Turkey. (oarikan@ee. bilkent.edu.tr)

S. Salous, Department of Electrical Engineering and Electronics, University of Manchester, P. O. Box 88, Manchester, M60 1QD, England, UK. (salous@umist.ac.uk) 